An evaluation of the Gent and Gent-Gent material models using inflation of a plane membrane

Lei Zhou, Shibin Wang, Linan Li, Yibin Fu

PII: S0020-7403(18)31665-5

DOI: 10.1016/j.ijmecsci.2018.07.035

Reference: $\quad$ MS 4447

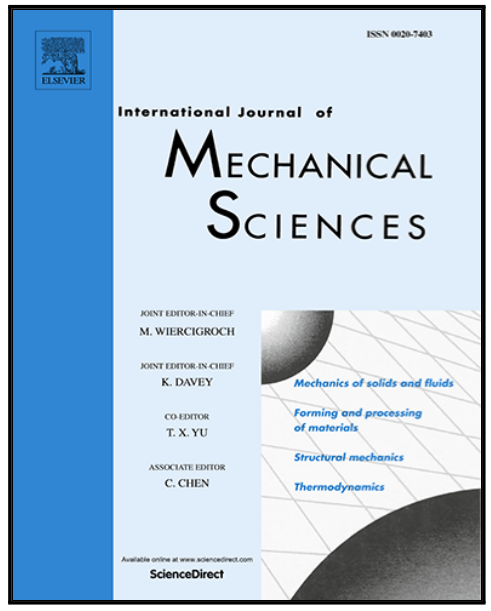

To appear in:

International Journal of Mechanical Sciences

Received date:

22 May 2018

Revised date:

21 July 2018

Accepted date:

25 July 2018

Please cite this article as: Lei Zhou, Shibin Wang, Linan Li, Yibin Fu, An evaluation of the Gent and Gent-Gent material models using inflation of a plane membrane, International Journal of Mechanical Sciences (2018), doi: 10.1016/j.ijmecsci.2018.07.035

This is a PDF file of an unedited manuscript that has been accepted for publication. As a service to our customers we are providing this early version of the manuscript. The manuscript will undergo copyediting, typesetting, and review of the resulting proof before it is published in its final form. Please note that during the production process errors may be discovered which could affect the content, and all legal disclaimers that apply to the journal pertain. 


\section{Graphical Abstract}

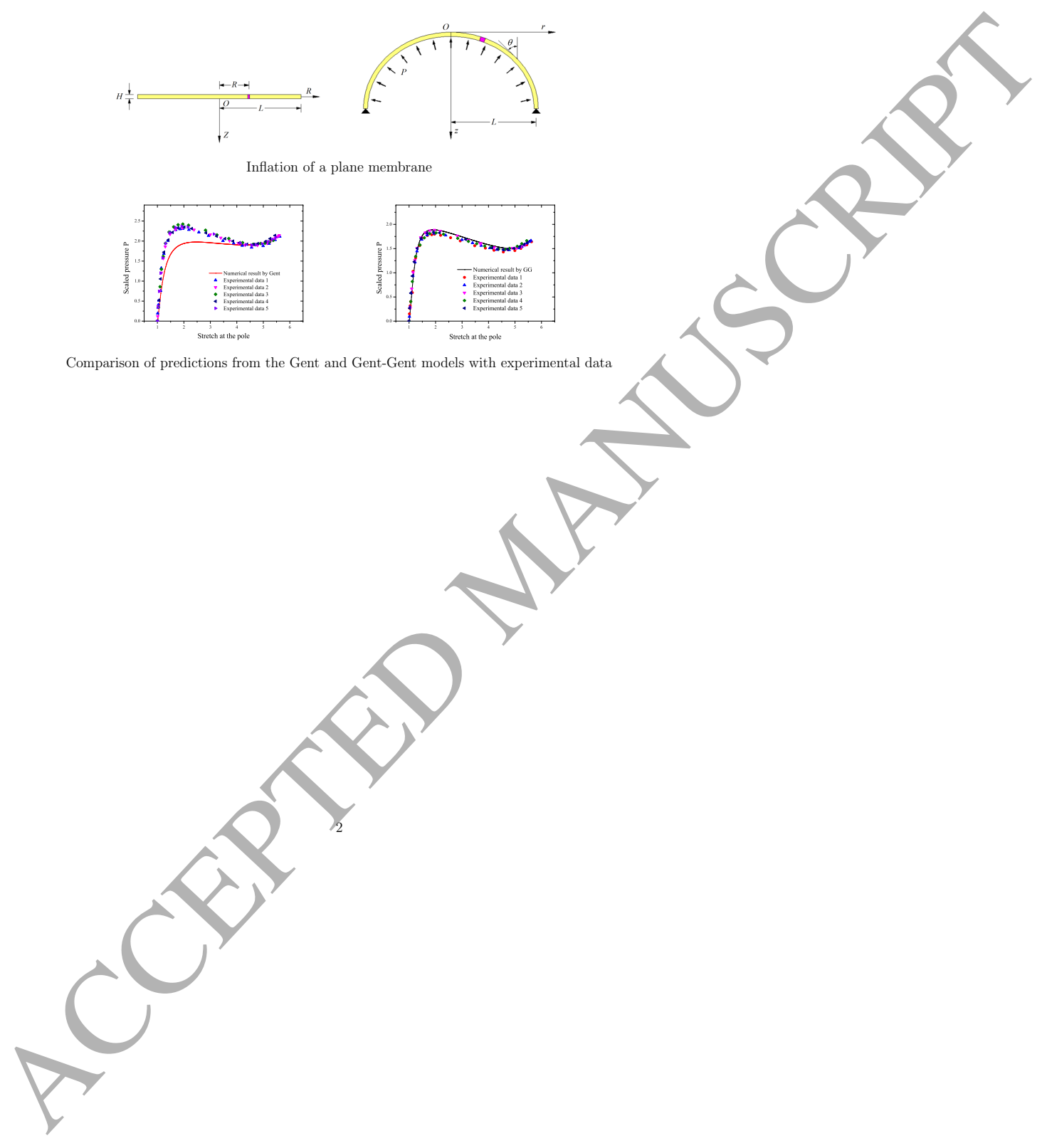




\section{Highlights}

- A new set of experiments is conducted on the inflation of plane membranes;

- The performance of Gent and Gent-Gent material models is evaluated, and it is shown that the Gent-Gent material model gives much better predictions than the original Gent material model;

- The accuracy of using inflation of a plane membrane to mimic equibiaxial extension is quantified.

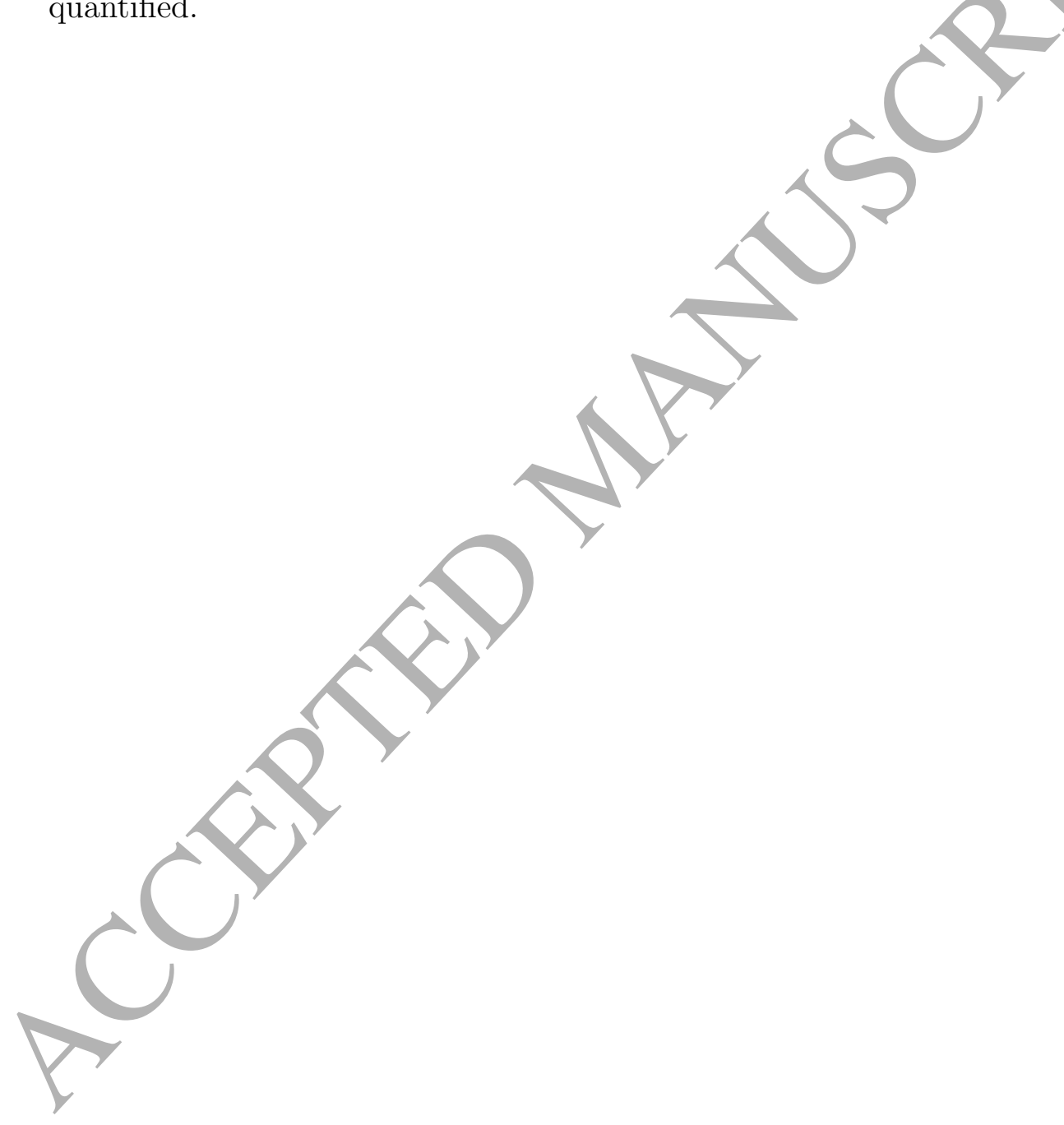




\title{
An evaluation of the Gent and Gent-Gent material models using inflation of a plane membrane
}

\author{
Lei Zhou ${ }^{[1]}$, Shibin Wang ${ }^{[1] *}$ Linan Li $^{[1]}$, Yibin Fu ${ }^{[1,2] \dagger}$ \\ ${ }^{1}$ Department of Mechanics, Tianjin University, Tianjin 300072, China \\ ${ }^{2}$ Department of Mathematics, Keele University, Staffordshire ST5 5BG, UK
}

\begin{abstract}
\end{abstract}
The Gent material model is the simplest extension of the neo-Hookean material model that can describe the finite extensibility of the polymeric chains comprising the elastomer network. However, it is known that its fitting to experimental results of uniaxial tension is not satisfactory for moderate values of stretch, and the GentGent model was proposed to remedy this deficiency. In this paper we provide further evidence on the good performance of the Gent-Gent model by using it to study the inflation of a circular plane membrane. For this problem, the deformation near the pole is equibiaxial and the associated nominal tension is a monotonic function of the stretch, but the pressure as a function of the stretch has both a maximum and a minimum. The Gent and Gent-Gent models are first fitted to our own experimental data for the nominal tension, and then used to predict variation of the pressure with respect to the stretch. By comparison with the experimental data, it is shown that the Gent-Gent model gives much better predictions than the Gent model.

keywords: Nonlinear elasticity, constitutive model, bulging, Gent model, Membrane

\section{Introduction}

Rubber-like material is a type of organic macromolecular polymeric material. Its complex molecular structure and ability to sustain large deformation requires a sophisticated nonlinear theory to characterize its mechanical properties. We refer to $[2,22]$ for a comprehensive review of the many constitutive models that have been proposed in the literature. Since all the constitutive models are necessarily approximate in nature, an important question

\footnotetext{
*Corresponding author at: shbwang@tju.edu.cn

†Corresponding author at: y.fu@keele.ac.uk
} 
to ask is how a material model will perform in more complex boundary value problems when the unknown parameters are fitted to experimental data from simple boundary value problems. The question is particularly pertinent if the material model is expected to give accurate quantitative predictions. However, there now exists an abundant literature in which approximate nonlinear constitutive models are unrealistically expected to give predictions that agree well with experimental results. In view of the increased use and adaption of the classical material models in the description of non-classical effects (e.g. those associated with electric and magnetic fields) [13, 14, 21, 25, 27], a more thorough examination of the performance of the classical models seems to be warranted.

Our current study focuses on the Gent material model that was proposed by Gent $[5,6]$ to model the rapid stiffening behaviour of rubber materials at large values of stretch, which is associated with the finite extensibility of the polymeric chains comprising the elastomer network. It has two parameters. One is related to the ground-state shear modulus, and the other one is a constant accounting for the extension limit. It is known that when the Gent model is fitted to experimental data of uniaxial tension, the fitting is usually good for small and large strains, but unsatisfactory for moderate values of strains. The reason for this characteristic was recognized by Pucci and Saccomandi [19] to be due to the fact that the Gent model predicts a Mooney plot that is flat in the large strain regime, but the corresponding experimental data usually exhibit an upturn. To describe this upturn, Pucci and Saccomandi [19] proposed a simple modification of the Gent model, which is now known as the Gent-Gent model. The superiority of this improved model has been discussed in $[16,18,19]$. The aim of this paper is to provide further evidence by revisiting the classical problem of the inflation of a circular plane membrane. We pose and answer the following question: if the material parameters are fitted to the experimental data for the nominal tension, how accurately will each model predict the variation of the inflation pressure as a function of the stretch? This is a non-trivial question because the pressure is not monotonic as pointed out in the Abstract, and good fitting to a monotonic tension curve does not necessarily imply good fitting to a non-monotonic pressure curve. This question is important when, for instance, we wish to accurately predict the initiation pressure at which localized bulging takes place in an inflated rubber tube $[3,4]$.

The boundary value problem associated with the inflation problem of a plane circular membrane was first formulated by Adkins and Rivlin [1] in 1952 and solved in a most elegant manner by a combination of Taylor expansion and scaling argument. Their formulation is valid for a general strain-energy function although their numerical calculations were conducted for the neo-Hookean and Mooney-Rivlin materials. Subsequent studies by Klingbeil and Shield [11] and Hart-Smith and Crisp [9] considered other material models in order to achieve better agreement with the experimental data of Treloar [23, 24]. Schmidt [21] 
carried out biaxial stretching of heat-softened plastic sheets, and multiple configurations were measured and analyzed. By comparing experimental data with those from the numerical solution, agreement over all configurations was about \pm 89.2 percent for the best models considered. Yang [28] showed that the governing equations can be rewritten as a system of three first order ordinary differential equations, which has since been followed by all subsequent investigations of inflation of plane, spherical, and ellipsoidal membranes $[4,20]$. More recently, the inflation problem has been studied under the additional effect of electric actuation $[7,8,10,25]$ motivated by increased use of inflated dielectric membranes in actuators.

Experiments on the inflation of a circular membrane were first conducted by Treloar $[23,24]$ who obtained the strain distribution and membrane shape at different stages of the inflation process. He also recognized the fact that in a sufficiently small neighbourhood of the pole, the deformation can be taken to be equibiaxial, and over a larger neighbourhood of the pole the inflated membrane can be viewed to be spherical. This set of experimental data is frequently used to calibrate material parameters in newly proposed material models. In this paper, we conduct our own experiments, and quantify the above facts observed by Treloar more precisely with the use of much better computing tools that now exist.

The rest of this paper is organized as follows. In the next section we summarize the governing equations and explain the procedure for determining the deformed configuration. In Sections 3 and 4 we present our experimental results, discuss the fitting of the Gent and Gent-Gent material models to the experimental data, and compare the theoretical predictions for the inflation pressure with experimental results. In our experiments, we also make use of assumptions that the deformation in a sufficiently small neighbourhood of the pole is homogeneous and equibiaxial, and that deformed membrane in a larger neighbourhood of the pole is spherical. These two assumptions are validated in Section 5. In the final section, we summarize our main results and conclude the paper with some additional remarks.

\section{Problem formulation and determination of the bulging solution}

We consider the inflation of a circular membrane that initially has thickness $H$ and radius $L$. This can be realized, for instance, by mounting the membrane on a cylindrical tube which contains compressed air. Since the profiles of the circular membrane before and after inflation are both axisymmetric, cylindrical polar coordinates $(R, Z)$ and $(r, z)$ are used to describe the position of material particles in the undeformed and deformed configurations, 
respectively; see Fig.1. We assume that the undeformed configuration is described by

$$
0 \leq R \leq L, \quad Z=0
$$

and the deformation is given by

$$
r=r(R), \quad z=z(R)
$$

We also introduce function $\theta(R)$ that denotes the angle between the tangent line and the vertical line at particle $R$ of the membrane; see Fig.1. This problem can be viewed a special case of the inflation of a full or truncated ellipsoidal membrane $[4,20,26]$.

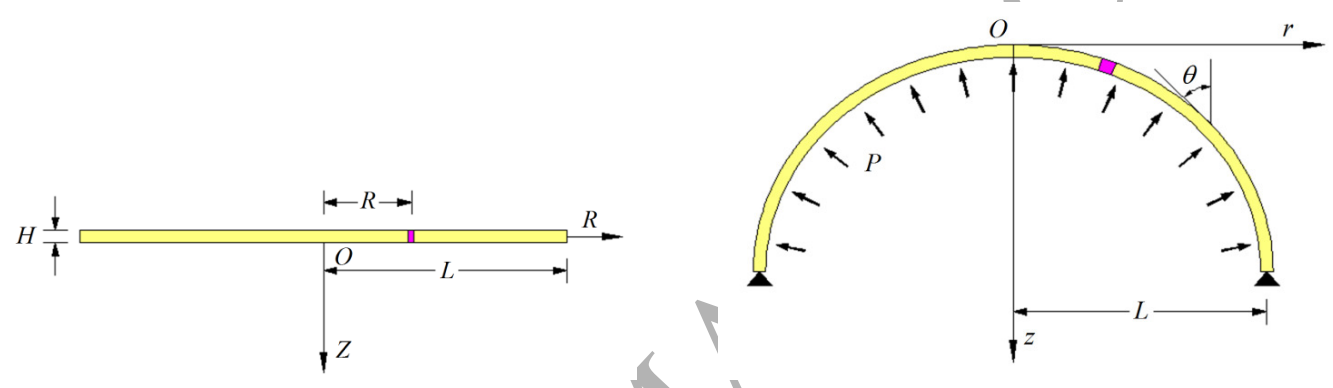

Figure 1: The undeformed (left) and deformed (right) configurations

The principal directions of stretch correspond to the directions of the latitude, the meridian and the normal to the deformed membrane. The associated principal stretches are given by

$$
\lambda_{1}=\frac{r}{R}, \quad \lambda_{2}=\sqrt{r^{\prime 2}+z^{\prime 2}}, \quad \lambda_{3}=\frac{h}{H},
$$

where $h$ is the deformed thicknesses and a prime denotes differentiation with respect to $R$. The material is assumed to be incompressible so that $\lambda_{1} \lambda_{2} \lambda_{3}=1$.

The total energy consists of the strain energy and the potential energy of the pressure $P$, and is given by

$$
E=\int_{0}^{L} W\left(\lambda_{1}, \lambda_{2}\right) 2 \pi R H \mathrm{~d} R-P \int_{0}^{L} \pi r^{2} z^{\prime} d R
$$

where $W\left(\lambda_{1}, \lambda_{2}\right)$ denotes the strain energy per unit volume in the undeformed configuration after $\lambda_{3}$ has been eliminated with the use of the relation $\lambda_{3}=1 /\left(\lambda_{1} \lambda_{2}\right)$.

The equilibrium equations can most conveniently be obtained by setting the first variation of $E$ to zero, and are given by

$$
r\left(h \sigma_{2}\right)^{\prime}+h r^{\prime}\left(\sigma_{2}-\sigma_{1}\right)=0
$$




$$
\frac{z^{\prime} \sigma_{1}}{r \lambda_{2}}+\frac{\sigma_{2}\left(r^{\prime} z^{\prime \prime}-r^{\prime \prime} z^{\prime}\right)}{\lambda_{2}^{3}}=\frac{\lambda_{1} \lambda_{2} P}{H}
$$

where $\sigma_{1}$ and $\sigma_{2}$ are the principal Cauchy stresses given by $\sigma_{1}=\lambda_{1} \partial W / \partial \lambda_{1}, \sigma_{2}=\lambda_{2} \partial W / \partial \lambda_{2}$. Equations (2.4) and (2.5) are equivalent to the equations (3.6) in Adkins and Rivlin [1] which are written in terms of the principal curvatures $\kappa_{1}=\cos \theta / r, \kappa_{2}=-\theta^{\prime} / \lambda_{2}=\left(r^{\prime} z^{\prime \prime}-r^{\prime \prime} z^{\prime}\right) / \lambda_{2}^{3}$.

With the use of the geometrical relations $r^{\prime}=\lambda_{2} \sin \theta, z^{\prime}=\lambda_{2} \cos \theta$, it is easy to show [28] that the two equilibrium equations (2.4) and (2.5) can be rewritten as the following system of first-order ordinary differential equations:

$$
\begin{aligned}
\frac{\mathrm{d} \lambda_{1}}{\mathrm{~d} R} & =\frac{\lambda_{2} \sin \theta-\lambda_{1}}{R}, \\
\frac{\mathrm{d} \lambda_{2}}{\mathrm{~d} R} & =\frac{W_{1}-\lambda_{2} W_{12}}{R W_{22}} \sin \theta-\frac{W_{2}-\lambda_{1} W_{12}}{R W_{22}}, \\
\frac{\mathrm{d} \theta}{\mathrm{d} R} & =\frac{W_{1}}{R W_{2}} \cos \theta-\frac{P \lambda_{1} \lambda_{2}}{H W_{2}} \\
\frac{\mathrm{d} z}{\mathrm{~d} R} & =\lambda_{2} \cos \theta,
\end{aligned}
$$

where $W_{1}=\partial W / \partial \lambda_{1}, W_{12}=\partial W^{2} / \partial \lambda_{1} \partial \lambda_{2}$, etc. We note that (2.9) is only needed if the variation of $z$ is required.

We non-dimensionalize our problem by making the substitutions

$$
(r, z, R) \rightarrow L(r, z, R),\left(W, \sigma_{1}, \sigma_{2}\right) \rightarrow \mu\left(W, \sigma_{1}, \sigma_{2}\right), \quad P \rightarrow \frac{\mu H}{L} P,
$$

where $\mu$ denotes the ground-state shear modulus. Under this substitution, the edge of the membrane corresponds to $R=1$.

At the pole of the deformed membrane, namely $R=0$, the axial symmetry requires that

$$
\lambda_{1}(0)=\lambda_{2}(0), \quad \theta(0)=\frac{\pi}{2}
$$

and

$$
\lambda_{1}^{\prime}(0)=\lambda_{2}^{\prime}(0)=\theta^{\prime \prime}(0)=0 .
$$

At the edge of the balloon, namely $R=1$, the fixed boundary implies that

$$
\lambda_{1}(1)=1
$$

The numerical solution of inflation is obtained by integrating the equations (2.6)-(2.9) subject to appropriate boundary conditions. We note that there exists a removable singularity at the pole $R=0$. We therefore integrate from $R=\delta$ to $R=1$ instead, where $\delta$ is a sufficiently small constant. We then need to obtain sufficiently accurate estimate for the values of $\lambda_{1}, \lambda_{2}$ and $\theta$ at $R=\delta$. 
On differentiating Eqs.(2.6)-(2.7) with respect to $R$ and evaluating at $R=0$, we can obtain two equations for $\lambda_{1}^{\prime \prime}$ and $\lambda_{2}^{\prime \prime}$, the solutions of which are given by

$$
\begin{aligned}
& \lambda_{1}^{\prime \prime}(0)=\frac{3 \lambda_{1} W_{11}-\lambda_{1} W_{12}+W_{1}}{8 W_{11}}\left(-\theta^{2}\right), \\
& \lambda_{2}^{\prime \prime}(0)=\frac{\lambda_{1} W_{11}-3 \lambda_{1} W_{12}+3 W_{1}}{8 W_{11}}\left(-\theta^{2}\right),
\end{aligned}
$$

where the right hand sides are evaluated at $R=0$. The $\theta^{\prime}(0)$ in the above expressions can be determined by evaluating (2.8) at $R=0$ and is given by

$$
\theta^{\prime}(0)=-\frac{P \lambda_{1}^{2}}{2 H W_{2}}
$$

where again the right hand side is evaluated at $R=0$. The expressions (2.14)-(2.16) can also be deduced from equations (7.15) and (7.16) in Adkins and Rivlin [1].

We may then write

$$
\begin{aligned}
& \lambda_{1}(\delta)=\lambda_{1}(0)+\frac{1}{2} \lambda_{1}^{\prime \prime}(0) \delta^{2} \pm O\left(\delta^{4}\right), \\
& \lambda_{2}(\delta)=\lambda_{2}(0)+\frac{1}{2} \lambda_{2}^{\prime \prime}(0) \delta^{2}+O\left(\delta^{4}\right), \\
& \theta(\delta)=\frac{\pi}{2}+\theta^{\prime}(0) \delta^{2}+O\left(\delta^{3}\right) .
\end{aligned}
$$

Thus, the initial values $\lambda_{1}(\delta), \lambda_{2}(\delta)$ and $\theta(\delta)$ are expressed in terms of the single unknown parameter $\lambda_{1}(0)$ (note that $\lambda_{2}(0)=\chi_{1}(0)$ ). Following Adkins and Rivlin [1], we solve the above boundary value problem using the following procedure: (1) Set $\lambda_{1}(0)$ at any desired value, and make a guess for the pressure $P$; $(2)$ The system of equations (2.6)-(2.8) are integrated from $R=\delta$ until $R=R_{0}$ say, where $\lambda_{1}\left(R_{0}\right)=1$; (3) The correct value of $P$ is then the above guessed yalue multiplied by $R_{0}$ because the system of equations (2.6)-(2.8) is invariant with respect to the substitutions $(R, P) \rightarrow\left(R / R_{0}, R_{0} P\right)$.

\section{Experiment}

In presenting our experimental results in this section, we suspend our non-dimensionalization (2.10). A single large latex rubber membrane sheet with thickness $0.35 \mathrm{~mm}$ was purchased from a commercial company. All the test sheets are cut out from this single membrane, and the test area of each rubber membrane has a radius of $45 \mathrm{~mm}$. The testing equipment is made up of three parts: (1) the inflation chamber (Fig.2) onto which the membrane was fixed and inflated into a bubble-like configuration, (2) the air pump that provided external pressure and permitted monitoring of the air velocity in real time, (3) measuring equipment and recording apparatus, including a multichannel recorder and a high speed camera which 
provided synchronized recording of the inflation pressure and bubble profile during bulging. We follow the standard protocol for experiments on rubber. In particular, our experiments were carried out at a room temperature of $23 \pm 2{ }^{\circ} \mathrm{C}$, and to minimize Mullins effect, the specimens were pre-inflated a number of times to the maximum stretch level reached in our actual experiments. It was observed that after each pre-inflation had been completed, there was some noticeable residual deformation and the membrane did not return to it plane form. The thickness at the centre reduced to $0.34 \mathrm{~mm}$, and there was a slight permanent bulging out. We thought that pre-stretching the plane membrane along two perpendicular directions in turn could reduce this permanent bulging out, but it turned out that pre-stretching made little difference. Therefore, the subsequent inflation experiment did not start from a plane circular plane exactly, as our theory part assumes. This is the major) source of our error. However, in our non-dimensionalisation, we do take the membrane thickness to be $0.34 \mathrm{~mm}$, rather than the original thickness before pre-inflation. Each inflation is completed in about 4 minutes.

In order to measure the stretch at the pole, before each experiment we mark the centre of the circular membrane and two points on a straight line through the centre which are both $4 \mathrm{~mm}$ from the center. The deformation in this small circular area of radius $4 \mathrm{~mm}$ is assumed to be homogeneous and equibiaxial. The three points in the inflated configuration are assumed to lie on a circle. Their coordinates are measured to $0.01 \mathrm{~mm}$, and used to compute the arc length and hence the stretch of the line segment. The accuracy of this approach to determine the stretch at the pole is assessed a posteriori in the penultimate section.

Besides the stretch, the internal pressure and the curvature at the pole are also recorded/measured. The radius of curvature could be determined from the three points described above, but for higher resolution it is better determined using two other points on a larger circle with radius $9 \mathrm{~mm}$. It is known that a larger neighbourhood of the pole can be assumed to be spherical than the neighbourhood where the deformation can be assumed to be homogeneous [23, 24]. The accuracy of this approach will also be quantified in the penultimate section.

Thus, the raw data obtained from the experiments include the coordinates of five marked points and the air pressure. The nominal stress $t_{\exp }$ is then determined from the following expression that is derived based on force balance in the polar area:

$$
t_{\exp }=\frac{\lambda_{2}(0) P}{2 H \kappa_{1}(0)}
$$

where $\kappa_{1}(0)$ is the curvature at the pole.

Fig. 3 shows the curvature determined by the method described above or by assuming the entire membrane to be spherical. In the latter method, the height of the (approximately) spherical balloon is measured and used to determine the curvature. As expected, the two 


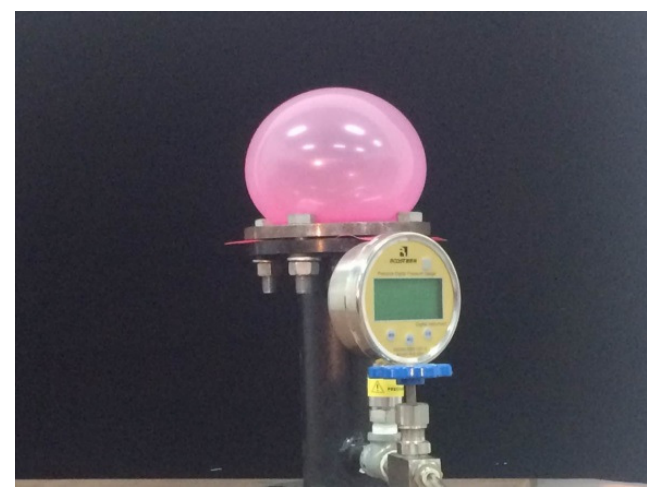

Figure 2: Experimental device for inflating a plane membrane

sets of results are close for small values of stretch, but they/differ more and more in the large stretch regime. When the stretch ratio is less than abøut 3.5, its relative error is less than $3 \%$, but as the stretch ratio exceeds 3.5 the membrane should no longer be viewed as being spherical.

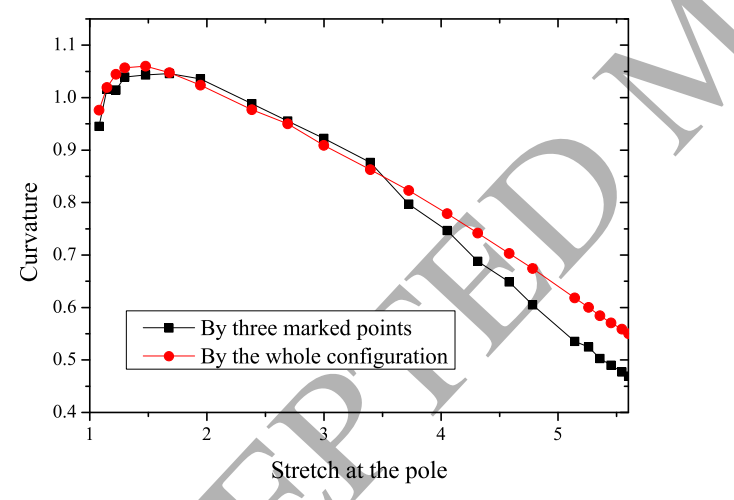

(a)

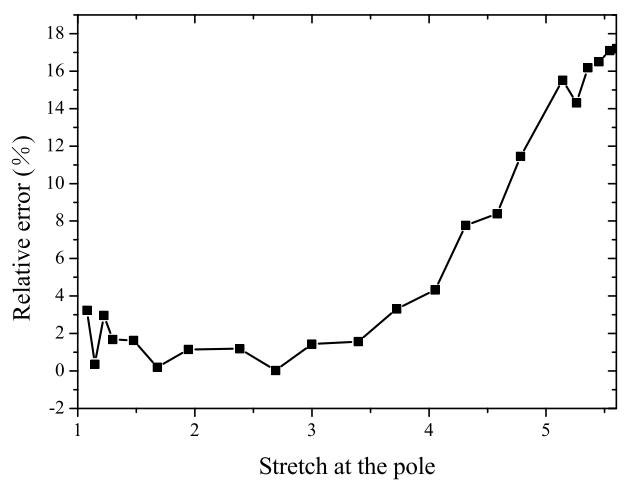

(b)

Figure 3: (a) Variation of curvature with respect to the stretch at the pole in the experiment, determined by the method described in the text (black line) or by assuming the entire configuration to be spherical (red line). (b) The relative error between the two different approaches.

Valid results are obtained for five circular membrane samples. Fig.4(a,b) shows that the whole experimental observation process is quite consistent. It is seen in Fig.4.(b) that the pressure curves exhibit the same $N$-shape as for most spherical balloons and cylindrical rubber tubes.

With the use of the above experimental data, the unknown parameters in any material model can be determined by the method of nonlinear least squares. In this work we consider 


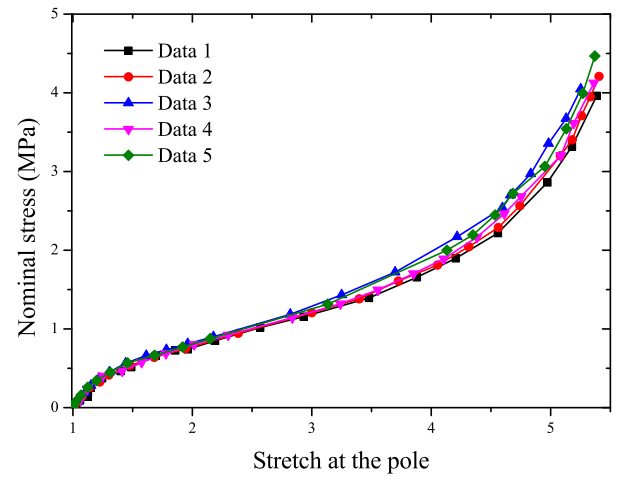

(a)

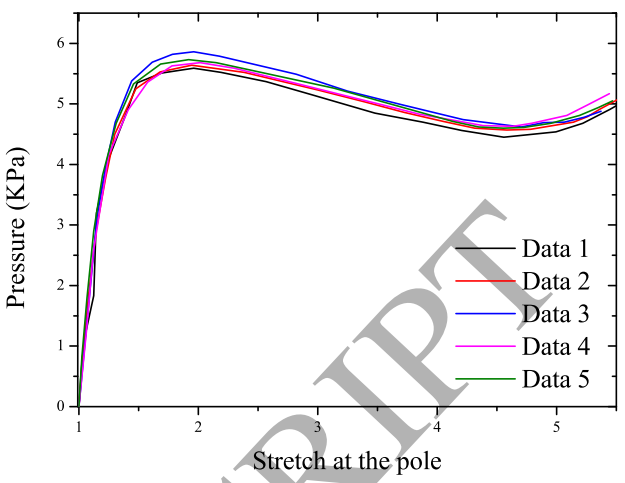

(b)

Figure 4: Five sets of experimental results for (a) the nominal stress at the pole as a function of the stretch at the pole, and (b) the pressure as a function of the stretch at the pole.

the Gent model [5, 6] and the Gent-Gent model (referred to as the GG model from now on) $[18,19]$ for the strain energy density function. We fit each model to all five sets of experimental data, and average the fitted parameter values to obtain the final result.

The Gent model has the form

$$
W=-\frac{\mu}{2} J_{m} \ln \left(1-\frac{I_{1}-3}{J_{m}}\right),
$$

where $I_{1}=\lambda_{1}^{2}+\lambda_{2}^{2}+\lambda_{3}^{2}, \mu$ denotes the ground-state shear modulus, and $J_{m}$ a material constant characterizing the extension limit.

For equibiaxial extension, we have $\lambda_{1}=\lambda_{2}=\lambda, \lambda_{3}=\lambda^{-2}$, and the nominal stress $t_{\text {fit }}$ is given by

$$
t_{\text {fit }}=\frac{\sigma_{1}}{\lambda_{1}}=-\frac{\mu J_{m}\left(\lambda^{6}-1\right)}{2 \lambda^{7}-\left(J_{m}+3\right) \lambda^{5}+\lambda}
$$

We denote the set of measured stretches by $\left\{\lambda^{(1)}, \lambda^{(2)}, \cdots, \lambda^{(m)}\right\}$, and the associated nominal stresses computed using (3.1) and (3.3) by $\left\{t_{\exp }^{(1)}, t_{\exp }^{(2)}, \cdots, t_{\exp }^{(m)}\right\}$ and $\left\{t_{\text {fit }}^{(1)}, t_{\text {fit }}^{(2)}, \cdots, t_{\text {fit }}^{(m)}\right\}$, respectively, where $m$ is the number of experimental data. The material parameters $\mu$ and $J_{m}$ are then determined by minimizing the error

$$
S=\sum_{i=1}^{m}\left(t_{\exp }^{(i)}-t_{\mathrm{fit}}^{(i)}\right)^{2} .
$$

The averaged parameter values thus determined are given by

$$
\mu=0.3200, \quad J_{m}=94.87, \quad S=0.1717,
$$



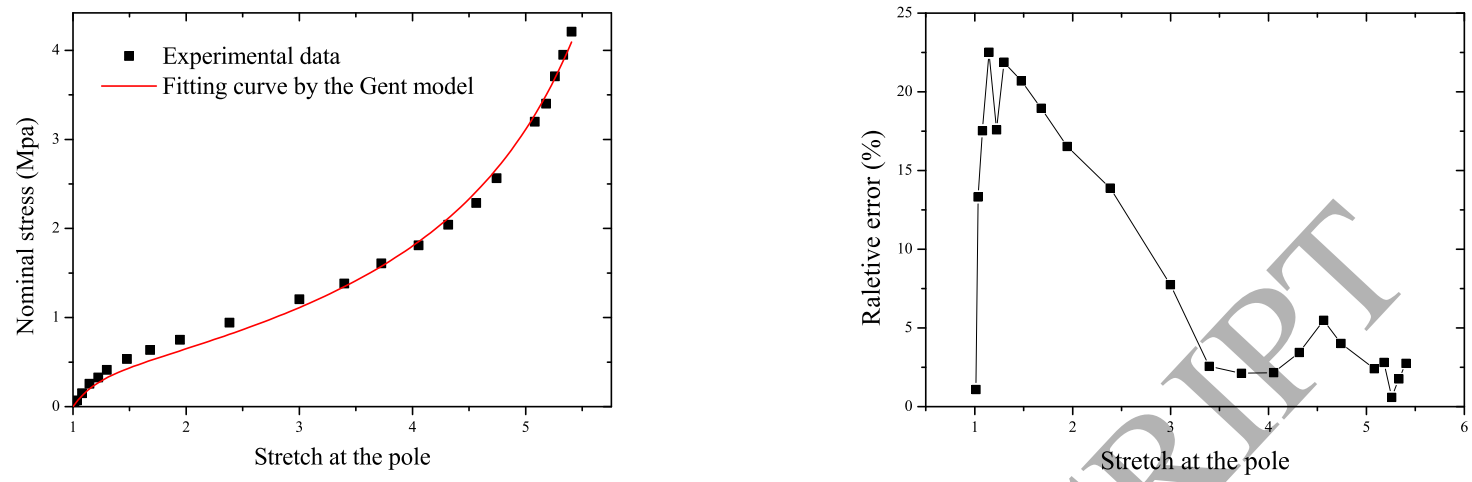

(a)

Figure 5: Fitting of the Gent model to experimental data of equibiaxial tension. (a) Variation of the nominal stress with respect to stretch. (b) Relative error of fitting.

and the fitting results for a typical set of experimental data are displayed in Fig.5.

The GG model has the form

$$
W=-\frac{\mu_{0}}{2} J_{m} \ln \left(1-\frac{I_{1}-3}{J_{m}}\right)+C_{2} \ln \frac{I_{2}}{3},
$$

where $I_{2}=\lambda_{1}^{-2}+\lambda_{2}^{-2}+\lambda_{3}^{-2}$, and $\mu_{0}, J_{m}$ and $C_{2}$ are material parameters. The ground-state shear modulus $\mu$ is given by $\mu=\mu_{0}+2 C_{2} / 3$.

The material parameters are fitted to the experimental data in a similar manner with the use of (3.1) and the following expression for the nominal stress:

$$
t_{\mathrm{fit}}=\frac{\sigma_{1}}{\lambda_{1}}=-\frac{\mu_{0} J_{m}\left(\lambda^{6}-1\right)}{2 \lambda^{7}-\left(J_{m}+3\right) \lambda^{5}+\lambda}+2 C_{2} \frac{\lambda^{6}-1}{\lambda^{7}+2 \lambda} .
$$

Table 1: The fitting result by the GG model

\begin{tabular}{ccccc}
\hline GG model & $\mu_{0}$ & $J_{m}$ & $C_{2}$ & $S$ \\
\hline Data 1 & 0.2672 & 87.08 & 0.1967 & 0.04422 \\
Data 2 & 0.2755 & 87.01 & 0.1904 & 0.04370 \\
Data 3 & 0.3026 & 90.40 & 0.1892 & 0.03702 \\
Data 4 & 0.2910 & 89.37 & 0.1695 & 0.03660 \\
Data 5 & 0.2903 & 88.29 & 0.2032 & 0.02260 \\
Averaged & 0.2853 & 88.43 & 0.1898 & 0.03683 \\
\hline
\end{tabular}

The fitted parameters associated with the five sets of experimental data together with their averaged values are given in Table 1, and the fitting results for a typical set of experimental data are displayed in Fig.6. The fitting results corresponding to the Gent model 

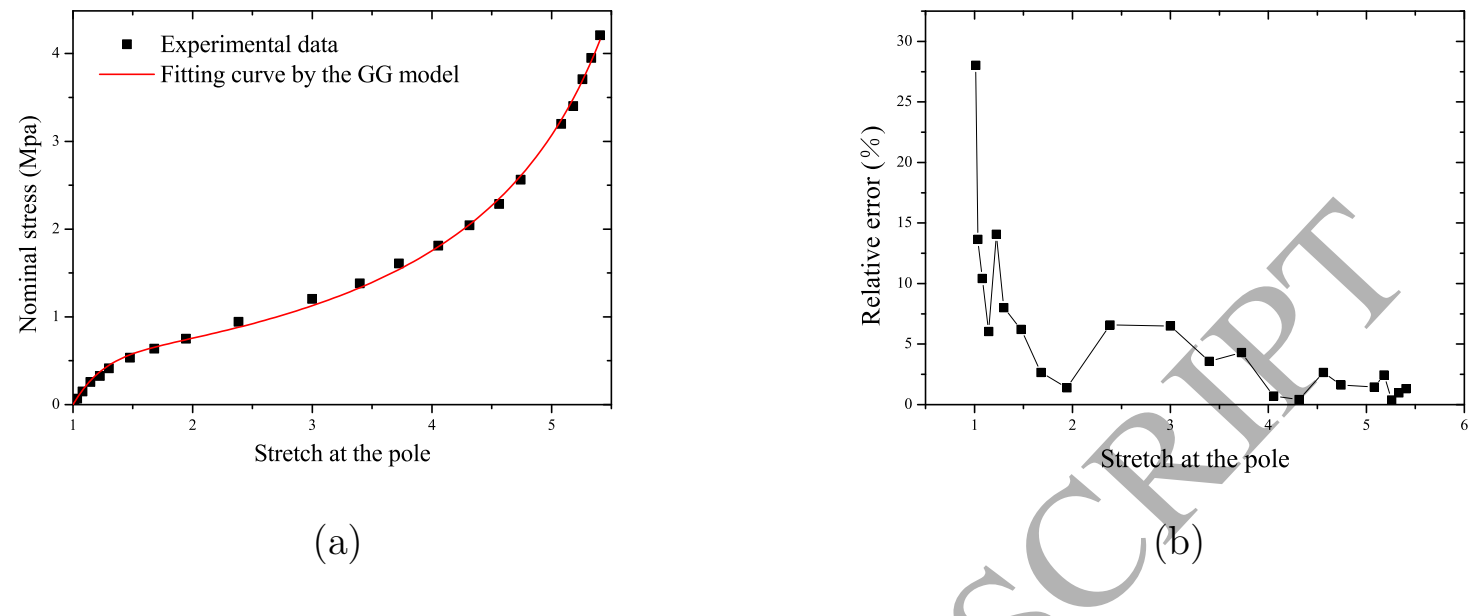

Figure 6: Fitting of the GG model to experimental data of equibiaxial tension. (a) Variation of the nominal stress with respect to stretch. (b) Relative error of fitting.

and the GG model are both seemingly acceptable. However, when the Gent model is used there are noticeable discrepancies in the moderate stretch regime between the fitting curve and experimental data. The maximum error reaches $23 \%$ even if the first five data are disregarded. In comparison, the fitting result corresponding to the GG model is much better: it can be seen in Fig.6(b) that the relative errors are below $7 \%$ when the first five data points are ignored. We also note that the $S$ value corresponding to the GG model is much smaller than that for the Gent model. Thus we conclude that the fitting curve by the GG model is in better agreement with the experimental data.

\section{Comparison of theoretical predictions with experi- mental results for the pressure}

In this section, we use the material models determined in the previous section to compute the pressure variation, and compare the results with the experimental data.

Fig.7 displays the predictions by the Gent and GG models, with the pressure maximum and minimum listed in Table 2. There is obvious difference between the predictions of the two models. To determine which prediction is better, in Fig. 8 we compare the individual predictions with the associated experimental results. The pressure determined from experiments has been scaled by $\mu H / L$ (see (2.10)), which takes different values for the two models used (since $\mu$ takes different values). It is seen that the GG model describes the experimental results much better than the Gent model, especially so near the pressure maximum. To highlight the good agreement between the predictions of the GG model and the experimental 
data, we have listed in Table 3 all the five sets of pressure maximum and minimum from experiments together with the theoretical predictions.

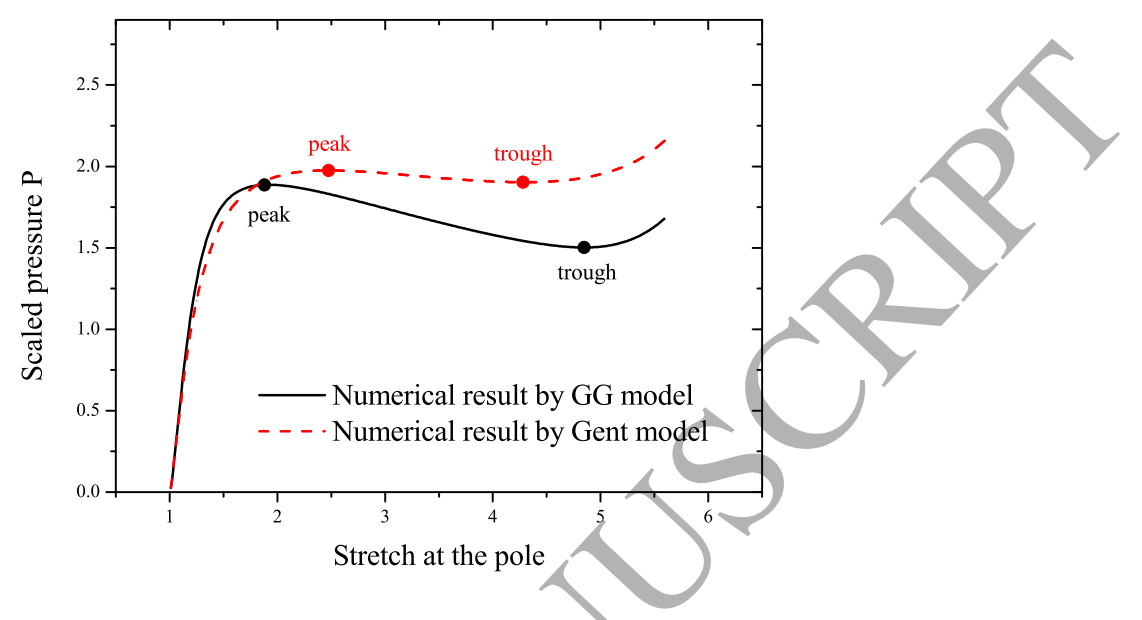

Figure 7: Theoretical predictions for the scaled pressure as a function of the stretch at the pole.

Table 2: Comparison of pressure maximum and minimum

\begin{tabular}{ccccc}
\hline Models & $\lambda$ (At the peak $)$ & $P($ At the peak $)$ & $\lambda($ At the trough $)$ & $P($ At the trough $)$ \\
\hline Gent model & 2.474 & 1.976 & 4.290 & 1.903 \\
GG model & 1.917 & 4.888 & 4.848 & 1.502 \\
\hline
\end{tabular}



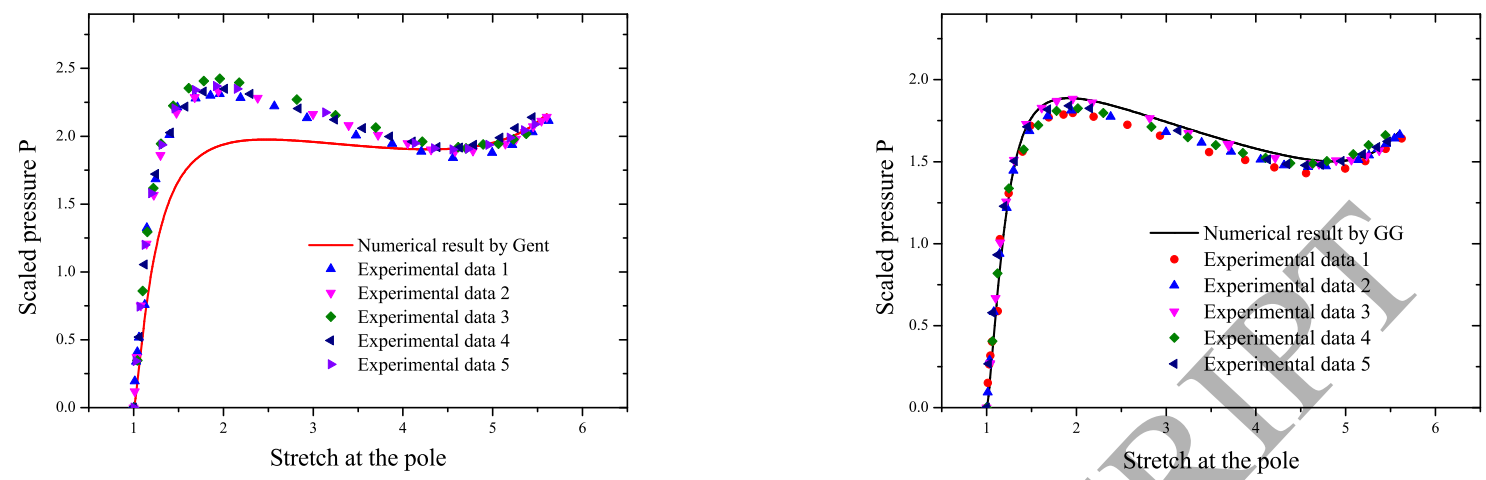

(a)

(b)

Figure 8: Comparison of experimental results with numerical results for the sealed pressure. (a) Gent model; (b) GG model. In each case, the numerical results are obtained by integrating the system of equations (2.6)(2.8) using the appropriate strain energy function.

Table 3: Pressure maximum and minimum and the associated values of stretch

\begin{tabular}{lcccc}
\hline Category & $\lambda($ At the peak $)$ & $P($ At the peak $)$ & $\lambda($ At the trough $)$ & $P($ At the trough $)$ \\
\hline Numerical calculation & 1.917 & 1.888 & 4.848 & 1.502 \\
Experimental data 1 & 1.960 & 1.796 & 4.558 & 1.430 \\
Experimental data 2 & $1.944)$ & 1.813 & 4.582 & 1.469 \\
Experimental data 3 & 1.960 & 1.883 & 4.704 & 1.485 \\
Experimental data 4 & 1.993 & 1.826 & 4.633 & 1.488 \\
Experimental data 5 & 1.919 & 1.841 & 4.554 & 1.478 \\
\hline
\end{tabular}

We next consider the sensitivity of the theoretical predictions to the variations of the material parameters in the GG model. We vary each material parameter above or below its original value by $10 \%$ and study the effect of each change on the pressure/stretch and stress/stretch curves.

We define a relative error $\xi$ through

$$
\xi=\frac{t_{c} / \mu_{c}-t_{o} / \mu_{o}}{t_{o} / \mu_{o}}
$$

where $t_{o}$ and $t_{c}$ denote the nominal stress based on the original values and changed values of material parameters, respectively, and $\mu_{o}$ and $\mu_{c}$ are the original and changed shear moduli, respectively. 


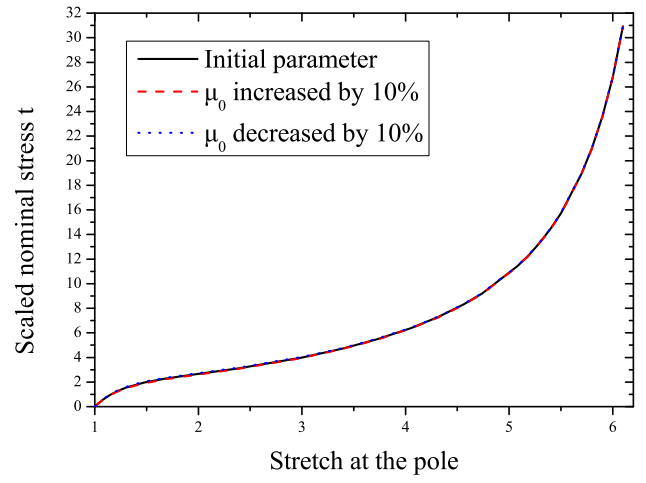

(a)

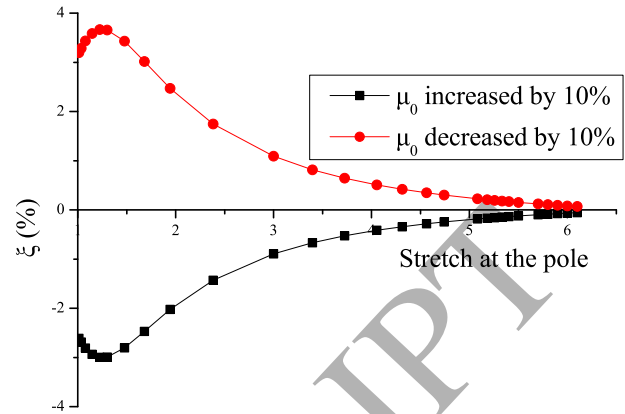

(b)

Figure 9: (a) Variation of the nominal stress with respect to the stretch when $\mu_{0}$ is increased or reduced by $10 \%$ from its original fitted value. (b) The relative error.

Figure 10: Variation of pressure with respect to the stretch when $\mu_{0}$ is increased or reduced by $10 \%$ from its original fitted value.

The results corresponding to changes in $\mu_{0}$ are shown in Figs 9 and 10. It can be seen that the greatest effects on the nominal stress and pressure are in the small-strain and large-strain regimes, respectively.

Figs 11 and 12 display the effects due to changes in $J_{m}$. It is seen that for both the nominal stress and pressure, the greatest effects are in the large strain regime, and the larger the stretch, the greater the effects. This is not surprising since $J_{m}$ characterizes the rapidly stiffening behavior of the rubber material. 


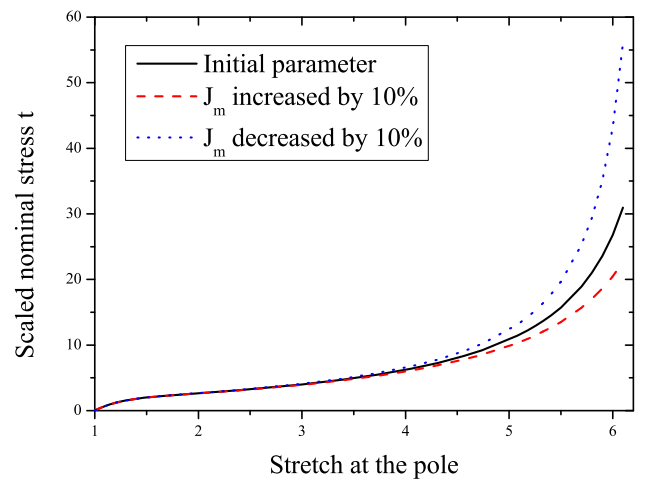

(a)

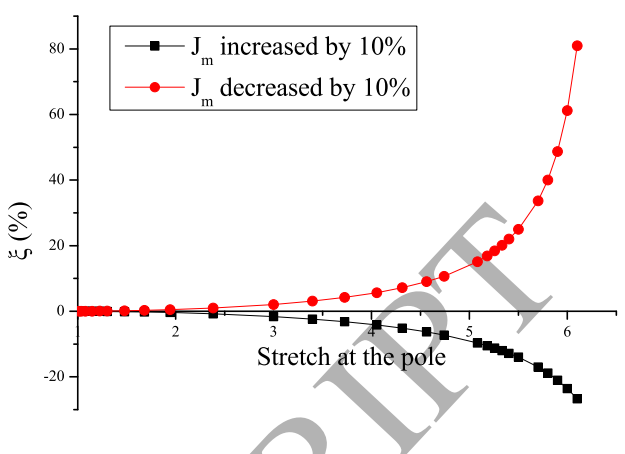

(b)

Figure 11: (a) Variation of the nominal stress with respect to the stretch when $J_{m}$ is increased or reduced by $10 \%$ from its original fitted value. (b) The relative error.

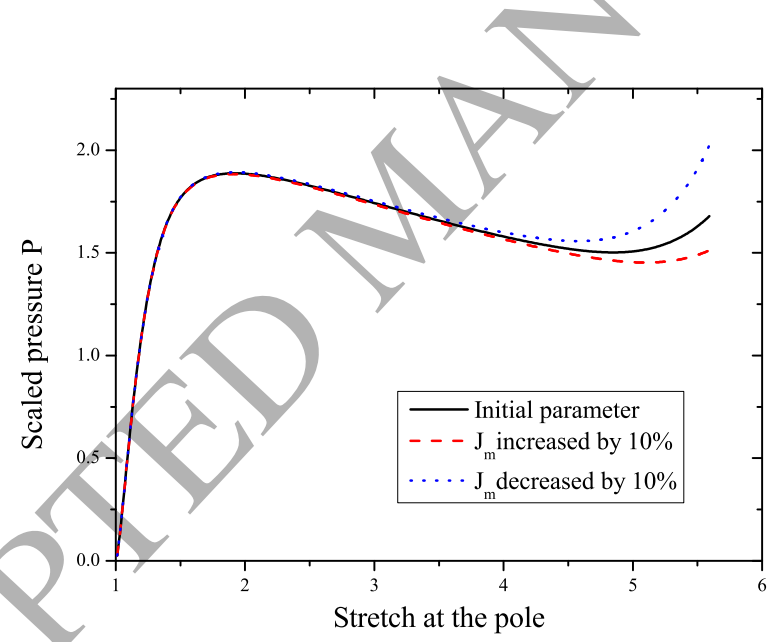

Figure 12: Variation of pressure with respect to the stretch when $J_{m}$ is increased or reduced by $10 \%$ from its original fitted value.

Finally, the effects due to changes in $C_{2}$ are shown in Figs 13 and 14. It is seen that the effects on the nominal stress are confined to the regime of small and moderate strains, and the effects on the pressure are confined to the regime of large strains. This is consistent with the known fact that adding the term involving $C_{2}$ improves data fitting in the regime of small and moderate strains. 


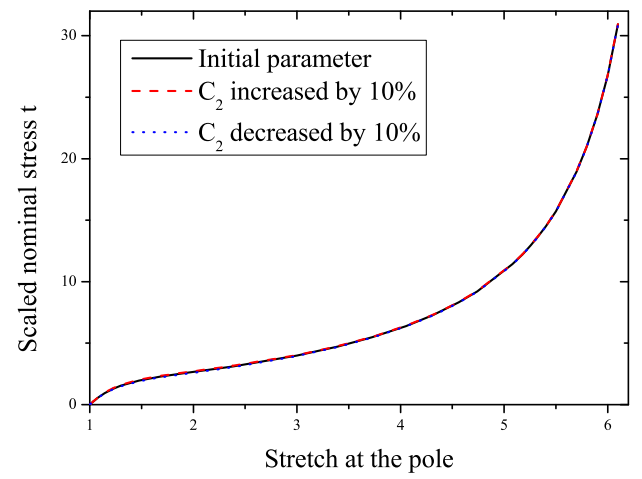

(a)

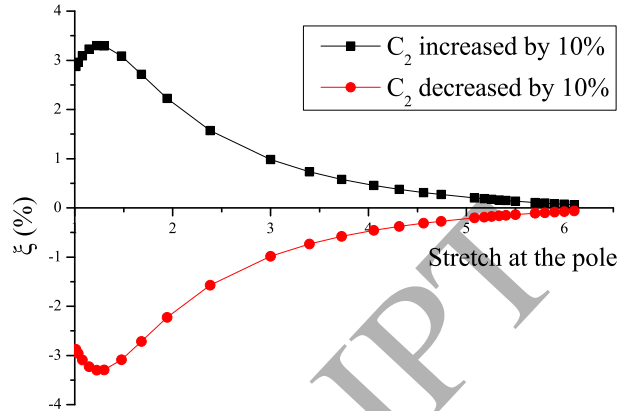

\section{2}

(b)

Figure 13: (a) Variation of the nominal stress with respect to the stretch when $C_{2}$ is increased or reduced by $10 \%$ from its original fitted value. (b) The relative error.

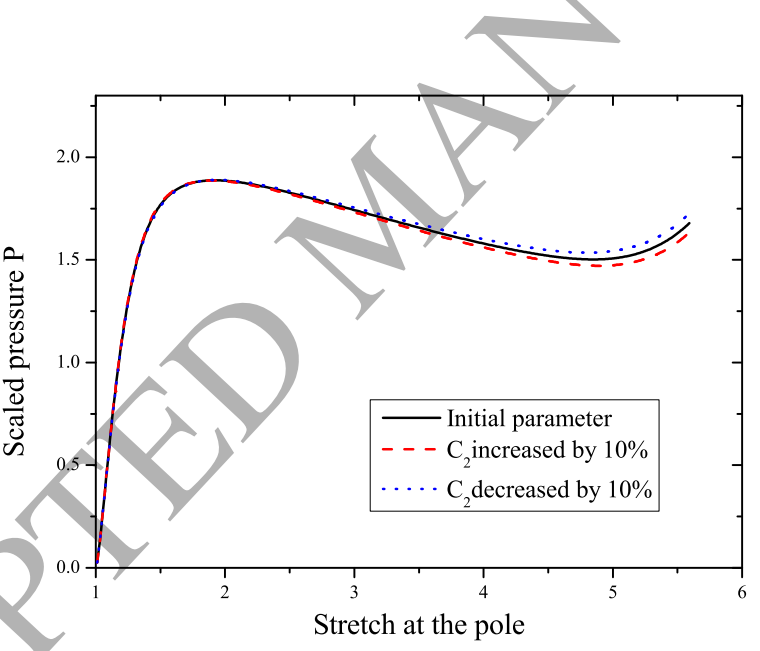

Figure 14: Variation of pressure with respect to the stretch when $C_{2}$ is increased or reduced by $10 \%$ from its original fitted value.

\section{Estimate of the accuracy of the assumptions made}

In this section, we provide a posteriori justification of our experimental method. We first quantify the error associated with the assumption that the deformation is equibiaxial in a small neighbourhood of the pole.

Fig.15(a) shows the variation of $\lambda_{1}$ and $\lambda_{2}$ with respect to $R$ corresponding to the four indicated values of $\lambda_{1}(0)$. It is seen that whereas $\lambda_{1}$ always decreases monotonically to its final value of 1 at the edge, the $\lambda_{2}$ may have a non-monotonic behaviour. This can be understood 

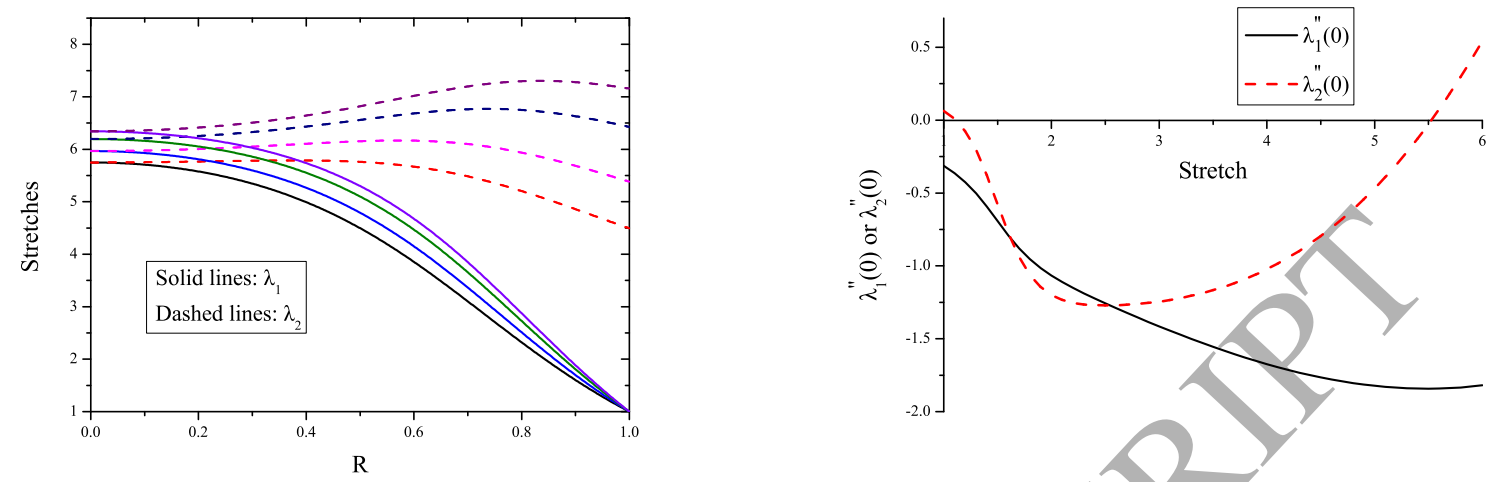

(a)

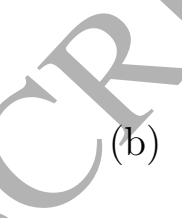

Figure 15: (a) Variation of $\lambda_{1}$ and $\lambda_{2}$ with respect to $R$ with $\lambda_{1}(0)=5.75,5.97,6.20,6.34$, respectively. (b) Variation of $\lambda_{1}^{\prime \prime}(0)$ and $\lambda_{2}^{\prime \prime}(0)$ with respect $\lambda_{1}(0)$.

by an inspection of the signs of $\lambda_{1}^{\prime \prime}(0)$ and $\lambda_{2}^{\prime \prime}(0)$, the variation of which with respect to $\lambda_{1}(0)$ is displayed in Fig.15(b). Whether $\lambda_{2}$ will increase or decrease in a neighbourhood starting from $R=0$ is determined by whether $\lambda_{2}^{\prime \prime}(0)$ is positive or negative [1]. Thus, we see that $\lambda_{2}$ will increase for small or large values of $\lambda_{1}(0)$, but will decrease for intermediate values of $\lambda_{1}(0)$.

In our experiment, a circle with non-dimensionalized diameter $2 R_{s}$ is marked with the pole as its center. If the arc length of the deformed diameter is $2 s$, then we have

$$
\lambda\left(R_{s}\right) \equiv \frac{s}{R_{s}}=\frac{1}{R_{s}} \int_{0}^{s} \mathrm{~d} s=\frac{1}{R_{s}} \int_{0}^{R_{s}} \lambda_{2} \mathrm{~d} R,
$$

where the first equation defines $\bar{\lambda}\left(R_{s}\right)$. The relative error when (5.1) is used to approximate the stretch at $R=0$ is then given by $1-\bar{\lambda}\left(R_{s}\right) / \lambda_{1}(0)$. Fig.16 shows how for some typical values of $R_{s}$ the relative error varies with respect to the stretch at the pole (i.e. $\lambda_{1}(0)$ ). It is seen that the maximum error occurs for intermediate values of the stretch at the pole. In our experiment, $R_{s}$ is taken to be $R_{s}=4 / 45=0.089$. The maximum error incurred is around $0.4 \%$. To keep the relative error to be below $1 \%$ or $5 \%$, the $R_{s}$ needs to be less than 0.14 or 0.31 , respectively.

We next assess the relative error associated with the estimation of the curvature that is used in (3.1) to compute the nominal stress. The two principal curvatures at $R=0$ are both equal to $-\theta^{\prime}(0) / \lambda_{2}(0)$ with $\theta^{\prime}(0)$ given by $(2.16)$. Thus, we have

$$
\kappa_{1}(0)=\kappa_{2}(0)=\frac{P \lambda_{1}(0)}{2 H W_{2}} .
$$

For each specified pressure $P$, the deformation can be obtained as illustrated in Section 3, and the associated value of $\lambda_{1}(0)$, and hence $\kappa_{1}(0)$ can be determined. However, in our 


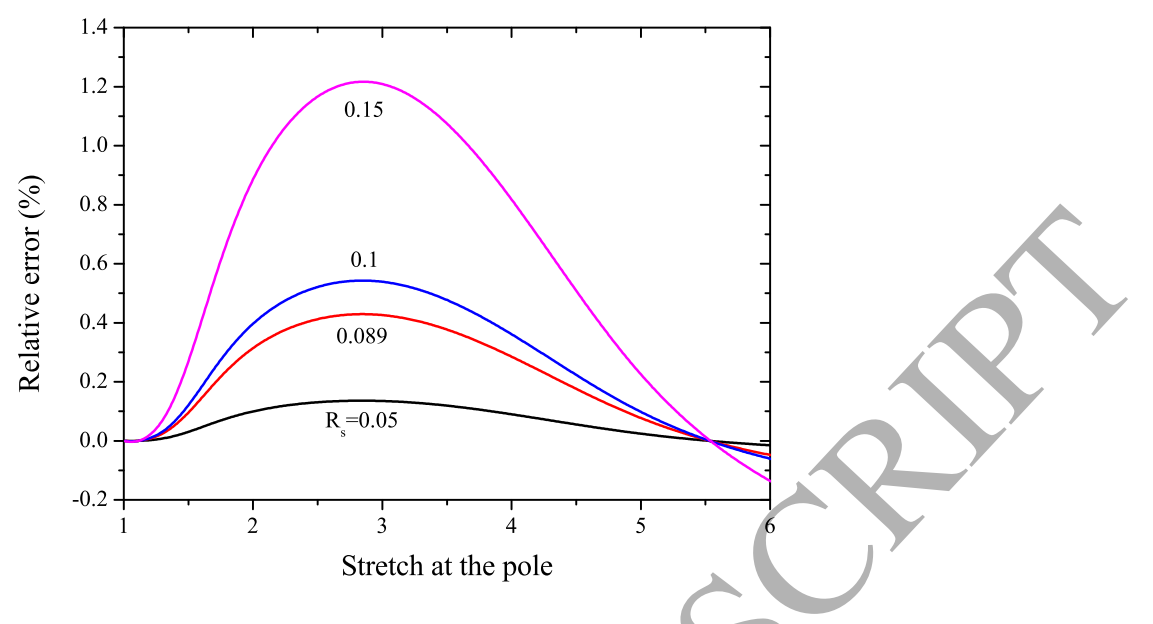

Figure 16: Dependence of relative error on $\lambda_{1}(0)$ for a selection of values of $R_{s}$.

experiments, this curvature value is approximated by the inverse of the radius of the circle going through the three points corresponding to $R=0, \pm R_{c}$, where $R_{c}$ is larger than the radius of the circle used to compute the stretch, and is taken to be 0.2 in our experiments. To assess the accuracy, we now take the coordinates of the three points to be given by our numerical solution and compute the approximate curvature. This approximate value is then compared with the exact value given by (5.2). The relative error as a function of the stretch at the pole for a selection of values of $R_{c}$ is displayed in Fig.17. It is seen that the largest errors are associated with the largest stretch, and when $R_{c}=0.2$ the relative error less than $1.7 \%$. To keep the relative error to be below $1 \%$ or $5 \%$, the $R_{c}$ needs to be less than 0.16 or 0.35 , respectively.

\section{Conclusion}

The inflation of a plane membrane is a classical problem, but as soft materials are increasingly used in high-tech applications, this problem is beginning to feature in a variety of situations under additional fields. For instance, dieletric membranes are increasingly used in various forms of actuators. Since many such actuators may operate on the verge of instability (pressure maximum) to maximize the actuated deformation [10], it is vitally important that the material models employed should be capable of predicting the maximum pressure accurately. In this paper, we have focussed on the purely mechanical case and shown that the Gent-Gent model is superior to the original Gent model in predicting the maximum and minimum pressure in the inflation of a plane membrane. We conducted our own experiments and recorded the variation of the tension at the pole and the inflation pressure with respect 


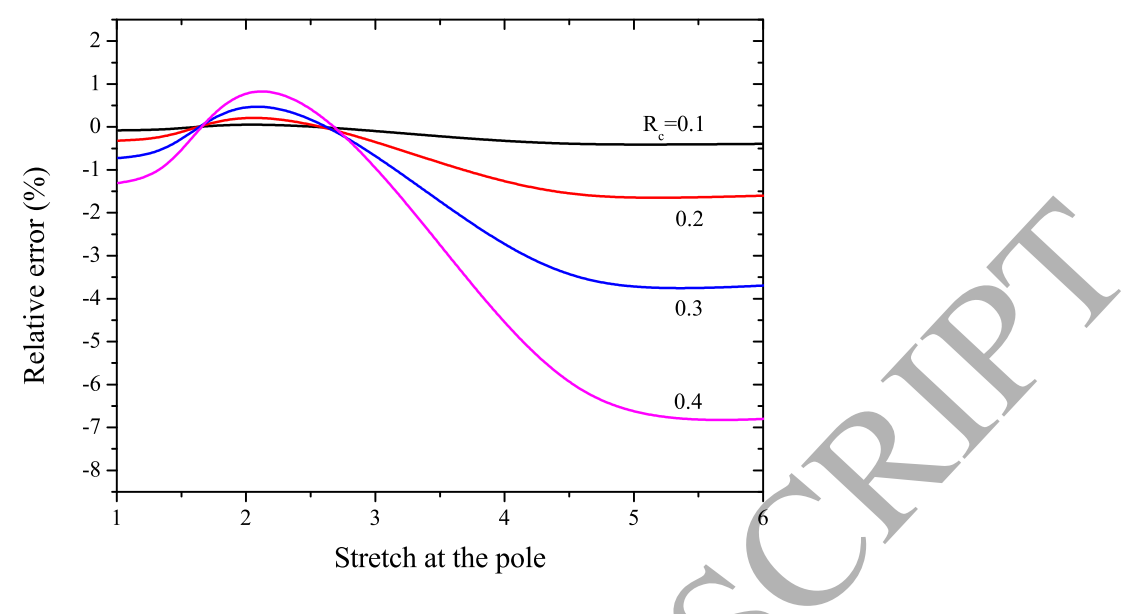

Figure 17: Dependence of relative error on $\lambda_{1}(0)$ for a selection of values of $R_{c}$.

to the stretch at the pole. The stretch at the pole is measured by following the deformation of a small circular area with the pole as its centre, and the curvature at the pole is estimated by making use of a larger circular area, as suggested by Treloar [24]. The errors incurred in the estimation of both quantities are estimated. It is shown that to keep the relative error below $1 \%$ the radii of the two circles should be less than 0.14 and 0.16 times the membrane radius, respectively, and to keep the relative error below $5 \%$ the radii of the two circles should be less than 0.31 and 0.35 times the membrane radius, respectively.

We remark that although we have focused on the Gent and Gent-Gent material models because of their simplicity (for instance, they are amenable to explicit analytic solutions for some basic problems of rubber elasticity involving nonhomogeneous deformations [12]), the methodology explained in the present paper can also be used to study other material models. In particular we have also studied the performance of the Ogden model [17] although the associated results are not presented for the sake of brevity. We have found that it performs as well as the Gent-Gent model despite the fact that an infinite number of solutions can be obtained for the material constants from data fitting by using different tolerance levels or initial guesses in the method of nonlinear least squares [18].

\section{Acknowledgements}

This work was supported by the National Natural Science Foundation of China (Grant No 11472186). 


\section{References}

[1] Adkins JE, Rivlin RS. Large elastic deformations of isotropic materials. ix. the deformation of thin shells. Philos T R Soc A 1952;244:505-31.

[2] Boyce MC, Arruda EM. Constitutive models of rubber elasticity: A review. Rubber Chem Technol 2000; 73:504-23.

[3] Fu YB, Pearce SP, Liu KK. Post-bifurcation analysis of a thin-walled hyperelastic tube under inflation. Int J Non-Linear Mech 2008;43:697-706.

[4] Geng YN, Huang JX, Fu YB. Shape bifurcation of a pressurized ellipsoidal balloon. Int J Eng Sci 2016; 101:115-25.

[5] Gent AN. Elastic instabilities in rubber. Int J Non-Linear Mech 2005; 40:165-75.

[6] Gent AN. A new constitutive relation for rubber. Rubber Chem Technol 1996; 69:59-61.

[7] Mockensturm EM, Goulbourne NC, Frecker MI. A nonlinear model for dielectric elastomer membranes. Journal of Applied Mechanics 2005; 72:899-906.

[8] Goulbourne NC, Mockensturm EM, Frecker MI. Electro-elastomers: Large deformation analysis of silicone membranes. Int J Splids Struct 2007; 44:2609-26.

[9] Hart-Smith LJ, Crisp JDC. Large elastic deformations of thin rubber membranes. Int J Eng Sci 1967; 5:1-24.

[10] Keplinger C, Li TF, Baumgartner R, Suo ZG, Bauer S. Harnessing snapthrough instability in soft dielectrics to achieve giant voltage-triggered deformation. Soft Matter $2012 ; 8: 285-8$.

[11] Klingbell WW, Shield RT. Some numerical investigations on empirical strain energy functions in the large axi-symmetric extensions of rubber membranes. ZAMP 1964; 15:608-29.

[12] Horgan CO, Saccomandi G. Phenomenological hyperelastic strain-stiffening constitutive models for rubber. Rubber Chem Technol 2006; 79:152-169.

[13] Liang XD, Cai SQ. Shape bifurcation of a spherical dielectric elastomer balloon under the actions of internal pressure and electric voltage. Journal of Applied Mechanics 2015; 82:504-23. 
[14] Lu TQ, Huang JS, Jordi C, Kovacs G, Huang R, Clarke DR, Suo ZG. Dielectric elastomer actuators under equal-biaxial forces, uniaxial forces, and uniaxial constraint of stiff fibers. Soft Matter 2012; 8:6167-73.

[15] Ma LS, Wang TJ. Nonlinear bending and post-buckling of a functionally graded circular plate under mechanical and thermal loadings. Int J Solids Struct 2003; 40:3311-30.

[16] Mangan R, Destrade M. Gent models for the inflation of spherical balloons. Int J Nonlinear Mech 2015; 68:52-58.

[17] Ogden RW. Large deformation isotropic elasticity: on the correlation of theory and experiment for incompressible rubberlike solids. Proc. R. Soc. Lond. A 1972; 326:565584.

[18] Ogden RW, Saccomandi G, Sgura I. Fitting hyperelastic models to experimental data. Comput Mech 2004; 34:484-502.

[19] Pucci E, Saccomandi G. A note on the gent model for rubber-like materials. Rubber Chem Technol 2002; 75:839-52.

[20] Sagiv A. Inflation of an axisymmetric membrane: Stress analysis. Journal of Applied Mechanics 1990; 57:682-687.

[21] Schmidt LR, Carley JF. Biaxial stretching of heat-softened plastic sheets using an inflation technique. Int J Eng Sci 1975; 13:563-78.

[22] Steinmann P, Hossain M, Possart G. Hyperelastic models for rubber-like materials: consistent tangent operators and suitability for treloars data. Arch Appl Mech 2012;82:1183217.

[23] Treloar LRG. Strains in an inflated rubber sheet and the mechanism of bursting. Rubber Chem Technol 1944;19:201-12.

[24] Treloar LRG. Stress-strain data for vulcanised rubber under various types of deformation. Trans Faraday Soc 1944;40:59-70.

[25] Wang FF, Yuan C, Lu TQ, Wang TJ. Anomalous bulging behaviors of a dielectric elastomer balloon under internal pressure and electric actuation. J Mech Phys Solids 2017;102:1-16.

[26] Wang T, Xu F, Huo Y, Potier-Ferry M, Snap-through instabilities of pressurized balloons: Pear-shaped bifurcation and localized bulging. Int J Non-linear Mech 2018; 98:137-44. 
[27] Xie YX, Liu JC, Fu YB. Bifurcation of a dielectric elastomer balloon under pressurized inflation and electric actuation. Int J Solids Struct 2016; 78:182-8.

[28] Yang WH, Feng WW. On axisymmetrical deformations of nonlinear membranes. J Appl Mech 1970; 37:1002-11. 